\title{
Reducing time spent by junior doctors on call performing routine tasks at weekends
}

\author{
Wadah Ibrahim, Simeng Lin
}

\begin{abstract}
At the Northern General Hospital, there are sixteen medical wards, spread over approximately half a mile. Weekend care for inpatients on these wards is provided by a team of four junior doctors, of different levels of training. We undertook a quality improvement project to reduce the amount of time junior doctors spent performing routine tasks at weekends. This may increase their available time for direct patient care.

The study was performed over a period of nine weeks on two medical wards - Diabetes \& Endocrine (W1) and Care of the Elderly Rehabilitation (W2). We monitored the bleeps received by the covering junior doctors during the weekend daytime shifts from the two study wards. We noted that a proportion of bleeps were routine tasks that could have been performed during weekday working hours. We also noted that W2 recorded fewer bleeps than W1 ward. This seemed to be because W2 batched junior doctors' jobs together. Firstly, we attempted to reduce the amount of routine work left undone each weekend. We provided a poster to remind Junior Doctors to complete such work during the week. Secondly, on W1 we replicated the job-batching system already in place on W2. A Doctors' Book was introduced in which nursing staff recorded the tasks that needed doing. This saved them from having to bleep the doctor repeatedly. The two changes resulted in a reduction in the number of bleeps generated by each ward and the number of visits required by the Junior Doctors to W1.
\end{abstract}

Simple changes can reduce the amount of time junior doctors spend performing routine work at weekends. We implemented two such changes and achieved a reduction in the number of bleeps experienced by junior doctors and the number of times they had to return to one ward.

\section{Problem}

We felt that when we worked on the weekend on-call cover team, a lot of time was spent responding to bleeps requesting work that should have been completed within normal working hours. These actions included such tasks as re-writing expired drug cards and prescribing IV fluids, warfarin and laxatives. Not only did this work reduce our time available for direct patient care but it also meant that nursing staff were spending time bleeping us or waiting for a response to bleeps. In addition, the necessity to perform this sort of work at weekends suggested that patients were not getting all their routine care in a timely manner.

\section{Background}

It is well-recognised that doctors often have to interrupt patient care to answer a bleep. It has also been demonstrated that a large proportion of those bleeps are not urgent and may even be inappropriate and/or incorrect. A group in Dublin found that $40 \%$ of bleeps to Junior Doctors were inappropriate or unnecessary in some way (1) whilst an American study documented that approximately $50 \%$ of bleeps interrupted doctors' patient care time (2). It isn't only the doctors' time being wasted. Nurses also have to interrupt their work to bleep doctors and await a response. A study in 2005 showed that, overall, nurses spent $8 \%$ of their time on the telephone. This varied according to shift. Nurses on the late shift spent nearly $18 \%$ of their time on the telephone (3). Furthermore, our Junior Doctors have to cover a wide physical area. Half a mile separates the furthest two wards and it takes ten minutes to walk from one to the other. It can be easily seen that returning to a ward to perform work that could have been done on a previous visit is not the most effective use of time.

\section{Baseline Measurement}

First, we needed to assess the size and nature of the problem. To do this, we made some baseline measurements on two of our acute medical wards. We elected to record the number of bleeps received by the on-call doctor in the 12 hour daytime shift on Saturday and Sunday. Both are medical wards, one is a Diabetic \& Endocrine ward (W1) and the other is Care of the Elderly Rehabilitation ward (W2). Both have 28 beds and both accept patients from the hospital's three Medical Assessment Units.

\section{We measured:}

- Number of bleeps from each ward in both weekend 12 hour daytime shifts (via switchboard records) for three weekends.

- The reason for the calls (from a nursing staff log).

Results showed that more bleeps originated from W1 than W2. In addition, there were more requests for routine work from $W 1$ than W2. This seemed to be because W2 batched Junior Doctors' tasks together, rather than issuing a bleep for each individual request.

See supplementary file: ds1877.xls - "Baseline measurment" 


\section{Design}

The analysis of the baseline measures suggested two possible areas of fruitful work:

1. Reduce the amount of routine work left undone by weekday medical teams.

2. Improve the batching of jobs on W1.

Brainstorming sessions were held with various stakeholders quality improvement staff, nursing staff, junior and senior medical staff. A number of ideas were generated from which we selected two simple tests of change to try. These two ideas were implemented as two PDSAs. Our measures for improvement remained the number of bleeps issued by each ward and the reasons for each call.

\section{Strategy}

PDSA 1

- We designed an A4 poster to act as a visual reminder of the need to complete routine work before the weekend. This was discussed with the ward managers and approval was obtained to display the posters in the doctors' offices and the nurses' stations on each ward.

- The aim of the project and the purpose of the poster were explained to the junior doctors and nurses on both wards.

PDSA 2

- Our second PDSA tried to improve job-batching on W1. We decided to replicate the system already in place on W2 and implemented a Doctors' Book, i.e. a note book that contained requests for all the non-urgent tasks to be done by doctors. Requests were recorded by staff nurses during the day and the book was checked regularly by junior doctors on both wards.

- The idea was explained to staff nurses and junior doctors and was given the green light from both ward managers.

\section{Results}

Following the first intervention, the total number of bleeps fell for one weekend but climbed back to baseline levels by the next weekend. The number of inappropriate bleeps did not decrease at all. Ward staff commented that, after two weeks, the posters were 'just another piece of furniture' and that their effect was tailing off. Two of the posters went missing from one of the wards and were never found.

It seemed clear that this first test of change was not sufficient to produce a sustained change and so we implemented the second PDSA. After introducing the Doctors' Job Book on W1, the number of inappropriate bleeps fell below baseline and has stayed at this new level. The idea was widely accepted by staff and we received positive feedback from both wards. It was felt that the book reminded doctors to complete routine tasks and saved both nursing and medical staff time by reducing the need for repetitive calls to the ward.

Following the success of the second intervention, a meeting was arranged with junior doctors on both wards to explain and embed the work. An explanation of the Doctors' Book was also added to the wards' induction information for the benefit of future cohorts of junior doctors. We will revisit the wards in eight weeks to ensure that improvements are embedded with the staff.

See supplementary file: ds1879.xls - "Charts showing 1. the total number of bleeps from both wards over the study period. 2. Chart showing bleeps for routine work"

\section{Lessons and Limitations}

We have learned several lessons from carrying out our project:

1. Simple changes can result in improvement.

2. However, the process is iterative. More than one test may be necessary and repeated measurement is needed to demonstrate improvement.

3. Engagement and feedback from stakeholders is vital for the design and implementation of a successful project.

4. Team work is important. This project would probably not have been successful without the input of both clinical and improvement staff.

5. Close involvement is necessary in the early stages of a project, if it is not to falter.

6. We acknowledge our lack of balance measures for this project i.e. a measure to ensure that the changes did not also have unwanted results.

\section{Conclusion}

Reducing the time that Junior Doctors spend on routine tasks is a worthwhile goal of improvement work as it may increase the time available to both nursing and medical staff for direct patient care. Our implementation of two small changes resulted in such a reduction on two of our medical wards at the weekends. We feel that this project was simple and could be easily implemented in other wards and hospitals. Our challenge now is to ensure that the improvements we have seen are sustained and handed over to the next cohort of junior doctors.

\section{References}

1. Beary E. Appropriateness of Bleeps: An Audit of the Bleep System Over Two Week Period. Irish Medical Journal May 2013 Volume 106 Number 5

2. Blum NJ, Lieu TA. Interrupted care. The effects of paging on pediatric resident activities. Am J Dis Child. 1992 Jul;146:806-8

3. Thomas J, Davies C. The nursing shortage and the scope for reducing it by redefining their role. Health Services 
Management Research 18: 217-222

\section{Declaration of interests}

Nothing to declare

\section{Acknowledgements}

Huge thanks to Dr. Anna Girolami for the invaluable guidance and the inspiring remarks. Thanks are also extended to Dr. Sally

Davies, the entire team of service improvement at Sheffield Teaching Hospitals and the nursing and medical staff who have willingly implemented these changes so effectively despite working in an extremely challenging environment. 